\title{
THE SET OF SECOND ITERATES IS NOWHERE DENSE IN $C$
}

\author{
KÁROLY SIMON
}

(Communicated by R. Daniel Mauldin)

\begin{abstract}
Let $C$ denote the set of continuous functions mapping $[0,1]$ into itself endowed with sup norm. It is proved that the set $\{f \circ f: f \in C\}$ is nowhere dense in $C$.
\end{abstract}

Let $C$ denote the set of continuous functions mapping $[0,1]$ into itself endowed with sup norm. Humke and Laczkovich [1,2] investigated the structure of the set $W^{2}=\{f \circ f: f \in C\}$. They proved that $W^{2}$ is an analytic non-Borel subset of $C$. They also proved that $W^{2}$ is not everywhere dense in $C$. The author of this paper proved that $W^{2}$ is a set of first category [5] and of zero Wiener measure [4]. Our aim here is to prove the

Therem. $W^{2}$ is nowhere dense in $C$.

For $f \in C$ and $n \in \mathbb{N}$ we define $f^{n}(x)$ by induction:

$$
f^{n}(x)=f\left(f^{n-1}(x)\right) \text { with } f^{1}(x)=f(x) .
$$

For each $n \in \mathbb{N} P_{f}^{n}$ denotes the set of periodic points of $f$ with period $n$ and $\operatorname{Fix}\left(f^{n}\right)=\left\{x: f^{n}(x)=x\right\}$; then $P_{f}^{n}=\operatorname{Fix}\left(f^{n}\right) \backslash \bigcup_{k<n} \operatorname{Fix}\left(f^{k}\right)$. The closed interval with endpoints $a$ and $b$ will be denoted by $\langle a, b\rangle$ even if $b<a$. We put

$$
B(f, \varepsilon)=\left\{h \in C: \sup _{x \in[0,1]}|f(x)-h(x)|<\varepsilon\right\} .
$$

Proof of theorem. Put

$$
\begin{aligned}
& K=\{f \in C: \text { for every } \varepsilon>0, \text { there exists } \delta>0, g \in B(f, \varepsilon) \\
& \text { such that } \left.B(g, \delta) \cap W^{2}=\varnothing\right\} .
\end{aligned}
$$

Thus $K$ is the set of all functions $f$ such that $W^{2}$ is not dense in any neighbourhood of $f$. It is enough to show that $K=C$. For every $f \in C$ we define $D_{f}=\left\{x: f^{\prime}(x)\right.$ exists $\}$. Put

$$
\begin{aligned}
\mathrm{PL}=\{f \in C: f \text { is piecewise linear; } \\
\\
\left.\quad \operatorname{Fix}(f) \subset D_{f} \cap(0,1) \text {; for every } x \in D_{f}\left|f^{\prime}(x)\right|>2\right\} .
\end{aligned}
$$

Received by the editors March 7, 1989 and, in revised form, November 1, 1989. 1980 Mathematics Subject Classification (1985 Revision). Primary 54H20. 
It is easy to see that $\mathrm{PL}$ is dense in $C$. We show that $\mathrm{PL} \subset K$, from which it follows that $K=C$ since $K$ is closed in $C$. Let $f \in \mathrm{PL}$ and $\varepsilon>0$ be fixed. We show that there exists $\delta>0$ and there is $g \in B(f, \varepsilon)$ such that $B(g, \delta) \cap W^{2}=\varnothing$. Since $f \in \operatorname{PL} \operatorname{card}(\operatorname{Fix}(f))$ is odd, assume that $2 n+1=\operatorname{card}(\operatorname{Fix}(f))$. Furthermore let $q=p_{1} \cdot p_{2} \cdots \cdots p_{(n+1)}$ where $5<p_{1}<p_{2}<\cdots<p_{(n+1)}$ are prime numbers. Then $\operatorname{Fix}\left(f^{q}\right)$ is a finite set since $f \in \mathrm{PL}$. Write $m=\operatorname{card}\left(\operatorname{Fix}\left(f^{q}\right)-\operatorname{Fix}(f)\right)$.

Lemma 0. There exist positive numbers $\delta_{1}, \delta_{2}$ such that $\varepsilon / 2>\delta_{1}>\delta_{2}>0$ and

(i) if $x \in \operatorname{Fix}(f)$ and $|t-x|<\delta_{1}$ then $f^{\prime}(t)$ exists;

(ii) if $h \in B\left(f, \delta_{2}\right)$ then $\operatorname{Fix}\left(h^{q}\right) \subset \bigcup_{x \in \operatorname{Fix}\left(f^{q}\right)}\left(x-\delta_{1}, x+\delta_{1}\right)$;

$$
\text { (iii) } \left.\begin{array}{l}
h \in B\left(f, \delta_{2}\right), \\
x, y \in k \leq \operatorname{Fix}\left(f^{q}\right), \\
f^{l}(x) \neq f^{k}(y)
\end{array}\right\} \Rightarrow h^{l}\left(\left(x-\delta_{1}, x+\delta_{1}\right)\right) \cap h^{k}\left(\left(y-\delta_{1}, y+\delta_{1}\right)\right)=\varnothing
$$

Proof of Lemma 0 . Choose $0<\eta_{1}$ so that it satisfies the following conditions: $\eta_{1}<\varepsilon / 2 ; \eta_{1}<\min \left\{|x-y|: x, y \in \operatorname{Fix}\left(f^{q}\right), x \neq y\right\}$, and if $x \in \operatorname{Fix}(f)$ with $|t-x|<\eta_{1}$ then $t \in D_{f}$. This is possible because $f \in \mathrm{PL}$.

We define $0<\eta_{2}$ so that $\eta_{2}<\eta_{1} / 10$ and

$$
|x-y|<\eta_{2} \Rightarrow \max _{0 \leq i \leq q}\left|f^{i}(x)-f^{i}(y)\right|<\eta_{1} / 10
$$

for every $x, y \in[0,1]$. Put $\delta_{1}=\eta_{2}$ and define $0<\eta_{3}<\delta_{1}$ so that $\eta_{3} \leq$ $\min \left\{\left|f^{q}(y)-y\right|: y \in[0,1]\right.$ and $\left.\operatorname{dist}\left(y, \operatorname{Fix}\left(f^{q}\right)\right) \geq \delta_{1}\right\}$. We know that $\eta_{3}>0$, thus we can choose $0<\eta_{4}$ so that $\eta_{4}<\eta_{3}$ and

$$
h \in B\left(f, \eta_{4}\right) \Rightarrow \min \left\{\left|h^{q}(y)-y\right|: \operatorname{dist}\left(y, \operatorname{Fix}\left(f^{q}\right)\right) \geq \delta_{1}\right\}>\eta_{3} / 2 .
$$

Choose $0<\eta_{5}$ so that $\eta_{5}<\eta_{4}$ and if $h \in B\left(f, \eta_{5}\right)$ then $\max _{0 \leq i \leq q}\left\|f^{i}-h^{i}\right\|<$ $\eta_{4}$. We put $\delta_{2}=\eta_{5}$.

It is obvious that (i) is fulfilled and that $(*)$ implies (ii). To see that (iii) holds let $h, l, k, x, y$ satisfy the conditions listed in (iii). Let $z_{1} \in\left(x-\delta_{1}, x+\delta_{1}\right)$, $z_{2} \in\left(y-\delta_{1}, y+\delta_{1}\right)$ be arbitrary. We have to show that $h^{l}\left(z_{1}\right) \neq h^{k}\left(z_{2}\right)$. We have $\left|f^{l}(x)-f^{l}\left(z_{1}\right)\right|<\eta_{1} / 10$ and $\left|f^{k}(y)-f^{k}\left(z_{2}\right)\right|<\eta_{1} / 10$ from the definition of $\eta_{2}$. Since $f^{l}(x), f^{k}(y) \in \operatorname{Fix}\left(f^{q}\right)$ and $f^{l}(x) \neq f^{k}(y),\left|f^{l}(x)-f^{k}(y)\right|>\eta_{1}$ follows from the definition of $\eta_{1}$. Thus $\left|f^{l}\left(z_{1}\right)-f^{k}\left(z_{2}\right)\right|>8 \eta_{1} / 10$. We know that $\left|h^{l}\left(z_{1}\right)-f^{l}\left(z_{1}\right)\right|<\eta_{2}<\eta_{1} / 10$ and $\left|h^{k}\left(z_{2}\right)-f^{k}\left(z_{2}\right)\right|<\eta_{2}<\eta_{1} / 10$ from the definition of $\eta_{5}$. Thus $8 \eta_{1} / 10<\left|f^{l}\left(z_{1}\right)-f^{k}\left(z_{2}\right)\right|<\left|f^{l}\left(z_{1}\right)-h^{l}\left(z_{1}\right)\right|+$ $\left|h^{l}\left(z_{1}\right)-h^{k}\left(z_{2}\right)\right|+\left|h^{k}\left(z_{2}\right)-f^{k}\left(z_{2}\right)\right|<\eta_{1} / 10+\left|h^{l}\left(z_{1}\right)-h^{k}\left(z_{2}\right)\right|+\eta_{1} / 10$ whence $6 \eta_{1} / 10<\left|h^{l}\left(z_{1}\right)-h^{k}\left(z_{2}\right)\right|$, which completes the proof.

Let $\operatorname{Fix}(f)=\left\{a_{1}^{\prime}, b_{1}^{\prime}, a_{2}^{\prime}, b_{2}^{\prime}, \ldots, a_{n}^{\prime}, b_{n}^{\prime}, a_{(n+1)}^{\prime}\right\}$ where $a_{i}^{\prime}<b_{i}^{\prime}<a_{(i+1)}^{\prime}$ $(i=1, \ldots, n)$ and put $U_{i}=\left(a_{i}^{\prime}-\delta_{1}, a_{i}^{\prime}+\delta_{1}\right), V_{j}=\left(b_{j}^{\prime}-\delta_{1}, b_{j}^{\prime}+\delta_{1}\right)$ for 
every $i=1, \ldots, n+1, j=1, \ldots, n$. Furthermore let $\operatorname{Fix}\left(f^{q}\right) \backslash \operatorname{Fix}(f)=$ $\left\{c_{1}, \ldots, c_{m}\right\}$ and put $W_{k}=\left(c_{k}-\delta_{1}, c_{k}+\delta_{1}\right)$ for every $1 \leq k \leq m$.

\section{THE MAIN STEPS OF THE PROOF}

To facilitate the presentation we will briefly outline the elements involved in our proof. There are two basic steps.

Step 1. In order to define a function $g \in B(f, \varepsilon)$ and find a suitable $\delta>0$ so that $B(g, \delta) \cap W^{2}=\varnothing$ we will first describe a technique for construction of a function $\Phi_{(I)}^{p}$, for a preassigned $p>5$ and interval $I \subset[0,1]$. Then we pick intervals $I_{i} \subset U_{i}$ for $1 \leq i \leq n+1$. The function $g$ will differ from $f$ only on $\bigcup_{i=1}^{n+1} U_{i}$. On each $I_{i}$, it will be a special function ' $\Phi_{\left(I_{i}\right)}^{p_{i}}$ derived from the technique. On the remainder of each $U_{i} \backslash I_{i} \quad g$ will be defined so that

(i) it is continuous on $[0,1]$;

(ii) it has no odd order periodic points in $\bigcup_{i=1}^{n+1}\left(U_{i} \backslash I_{i}\right)$.

Then the $\delta$ is chosen so that any function $v \in B(g, \delta)$ will have at least $m+1$ periodic orbits of period $p_{i}$ in $U_{i}, 1 \leq i \leq n+1$.

Step 2. We will examine, via 4 lemmas, the possible locations of periodic orbits of period $p_{i}$ for any function $v \in B(g, \delta)$. It will be shown that the assumptions $v \in B(g, \delta)$ and $v=h^{2}$ for some $h \in C$ are self-contradictory.

\section{STEP 1}

Let $|I|$ denote the length of the interval $I$. Put $\gamma=|I| \cdot[2(2 p+1)]^{-1}$ and define the pairwise disjoint closed intervals

$$
A_{i}=\left[d_{i}-\gamma, d_{i}+\gamma\right] \quad(i=0,1,2, \ldots, p-1)
$$

where $d_{0}=\gamma, d_{i}=d_{(i-1)}+4 \gamma \quad 1 \leq i \leq p-2$, and $d_{(p-1)}=d_{(p-2)}+8 \gamma$. Write $d^{\prime}=d_{(p-2)}+4 \gamma$ and denote by $\Omega$ the increasing linear function mapping $I$ onto $\left[d^{\prime}-\gamma, d^{\prime}+\gamma\right]$. Next we define a function $\Phi^{p}$ on $[0,1]$ which is to be linear on the intervals $A_{i}(i=0,1, \ldots, p-1),\left[d^{\prime}-\gamma, d^{\prime}+\gamma\right]$ and on the intervals contiguous to them: put $\Phi^{p}\left(d_{i}-\gamma\right)=d_{(i+1)}-9 \gamma / 10, \Phi^{p}\left(d_{i}+\gamma\right)=d_{(i+1)}+9 \gamma / 10$ $(i=0,1, \ldots, p-2) \Phi^{p}\left(d_{(p-1)}-\gamma\right)=d_{0}+9 \gamma / 10, \Phi^{p}\left(d_{(p-1)}+\gamma\right)=d_{0}-9 \gamma / 10$.

We define $\gamma^{\prime}=\gamma /(2 p+1)$ from $\gamma^{\prime} /|\Omega([0,1])|=\gamma$ and put

$$
\Phi^{p}\left(d^{\prime}-\gamma\right)=d^{\prime}-\gamma+4.1 \gamma^{\prime}, \Phi^{p}\left(d^{\prime}+\gamma\right)=d^{\prime}-\gamma+0.1 \gamma^{\prime} .
$$

Thus we have defined $\Phi^{p}$ (see Figure 1, p. 1144). It is easy to see that $\Phi^{p}$ has the following properties:

(A) If $\left\|h-\Phi^{p}\right\|<\gamma \cdot(0.9)^{p} / 30$ then there exists a periodic orbit of $h$ with period $p$ in $\bigcup_{i=0}^{p-1}\left(d_{i}-\gamma / 3, d_{i}+\gamma / 3\right)$.

(B) If $\left\|h-\Phi^{p}\right\|<\gamma \cdot(0.9)^{p} / 30$ and $\left\{x_{i}\right\}_{i=0}^{p-1}$ is a periodic orbit of $h$ with period $p$ in $\bigcup_{i=0}^{p-1} A_{i}$ then $\left\{x_{i}\right\}_{i=0}^{p-1} \subset \bigcup_{i=0}^{p-1}\left(d_{i}-\gamma / 3, d_{i}+\gamma / 3\right)$. 


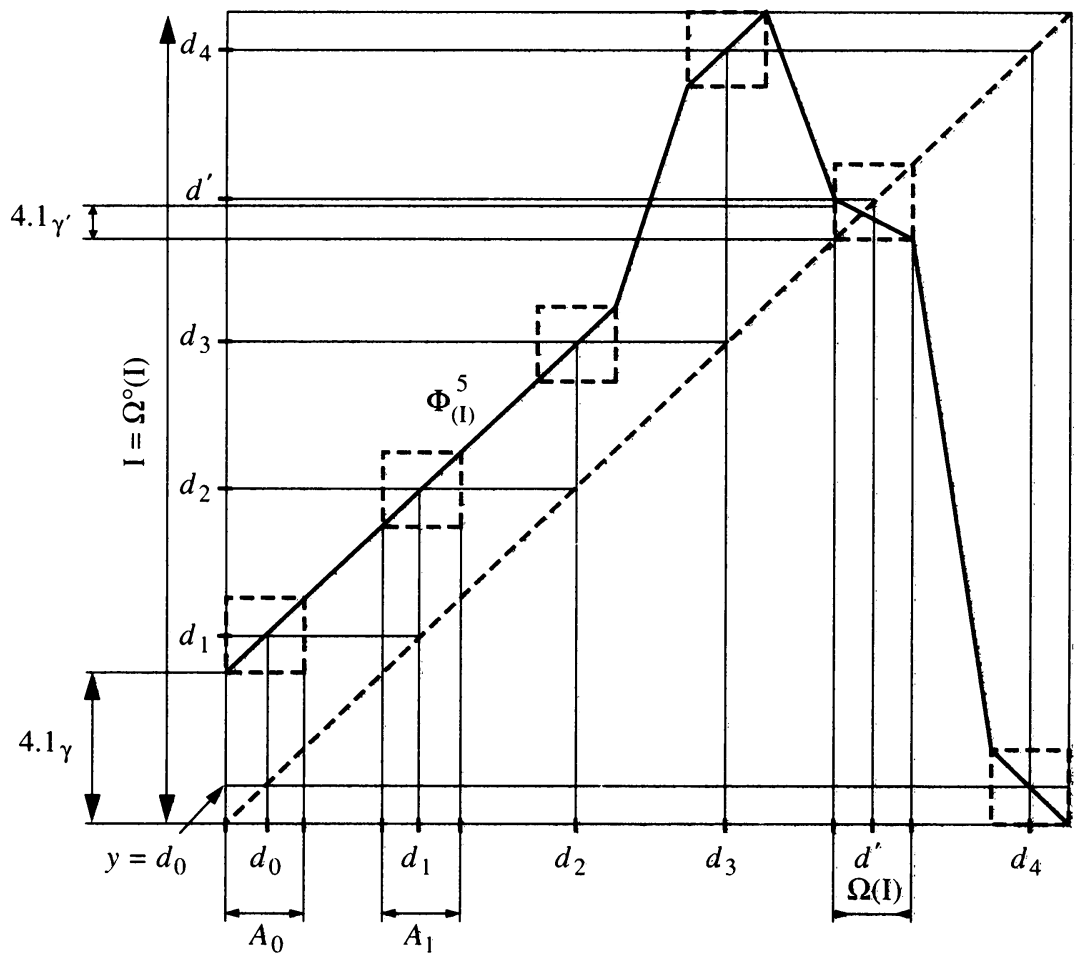

Figure 1

(C) If $\left\{x_{i}\right\}_{i=0}^{p-1} \subset I \backslash \Omega(I)$ is a periodic orbit of $\Phi^{p}$ and $p$ is odd then the smallest interval which contains $\left\{x_{i}\right\}_{i=0}^{p-1}$ also contains the point $d_{p-2}$ (see Figure 1).

Proof of (A) and (B). Let $d_{0}-\gamma<x \leq d_{0}-\gamma / 3$ and $d_{0}+\gamma / 3<y \leq d_{0}+\gamma$. Since the absolute value of the slope of $\Phi^{p}$ above $A_{i}(0 \leq i \leq p-1)$ equals to 0.9 it is implied that if $\left\|h-\Phi^{p}\right\|<\gamma \cdot(0.9)^{p} / 30$ then $h^{p}(x)>d_{0}$ and $h^{p}(y)<d_{0}$. So the interval $(x, y)$ contains a periodic point of $\Phi^{p}$ with period $p$ (this proves (A)), but $x, y$ are not periodic points of $\Phi^{p}$ with period $p$ so we have proved (B) as well.

Proof of $(\mathrm{C})$. The fact that $\left\{x_{i}\right\}_{i=0}^{p-1}$ is a periodic orbit of $\Phi^{p}$ implies that there exists $0 \leq 1 \leq p-1$ such that $\Phi^{p}\left(x_{l}\right)<x_{l}$ whence $x_{l}>d^{\prime}+\gamma$. Without loss of generality we may assume that $l=0$. If $x_{k}>d_{p-2}$ for every $0 \leq k \leq p-1$ then $x_{1}, x_{3}, \ldots, x_{p-2}, x_{0} \in\left[d_{p-2}+\gamma, d^{\prime}-\gamma\right]$ which contradicts the fact that $x_{0}>d^{\prime}+\gamma$ (see Figure 1).

For an arbitrary interval $I$ let $\tau_{I}$ denote the similarity transformation mapping $[0,1]$ onto $I$. We define

$$
\Phi_{(I)}^{p}(x)=\tau_{I}\left(\Phi^{p}\left(\tau_{I}^{-1}(x)\right)\right) \quad(x \in I) .
$$




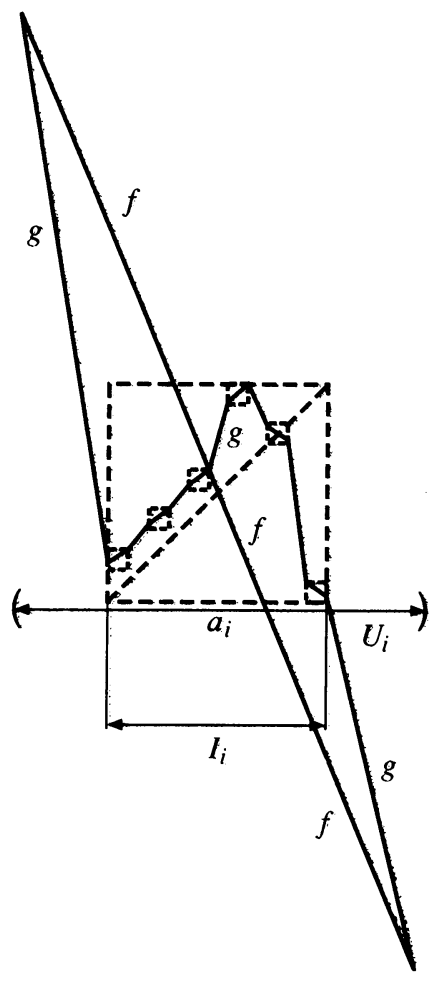

FIGURE 2

Put

$$
{ }^{\prime} \Phi_{(I)}^{p}(x)= \begin{cases}\Phi_{\Omega^{i}(I)}^{p}(x), & \text { if } x \in \Omega^{i}(I) \backslash \Omega^{i+1}(I) \text { and } 0 \leq i<m ; \\ \Phi_{\Omega^{m}(I)}^{p}(x), & \text { if } x \in \Omega^{m}(I) .\end{cases}
$$

We next define $g \in C$ as follows (see Figure 2). We put $g(x)=f(x)$ if $x \notin \bigcup_{i=1}^{n+1} U_{i}$.

Let $1 \leq i \leq n+1$ be fixed. We choose an interval $I_{i} \subset U_{i}$ with midpoint $a_{i}$ such that $\left|I_{i}\right|<\delta_{2} /\left(2\left|f^{\prime}\left(a_{i}\right)\right|\right)$. Let $g$ be linear decreasing on both components of $U_{i} \backslash I_{i}$ and

$$
g(x)={ }^{\prime} \Phi_{\left(I_{i}\right)}^{p}(x) \quad\left(x \in I_{i}\right) .
$$

Thus we have $g \in C \cap B\left(f, \delta_{2}\right)$. Put $\delta=\min _{1 \leq i \leq n+1}\left|\Omega^{m}\left(I_{i}\right)\right| / 100$. Let $1 \leq i \leq n+1$ be fixed and let

$$
A_{k}^{i}=\tau_{t_{i}}\left(A_{k}\right) \quad\left(k=0,1,2, \ldots, p_{i}-1\right) .
$$

Proposition 1. We can see that if $\|h-g\|<\delta$ then:

(a) For every $1 \leq i \leq n+1 \quad h$ has at least one periodic orbit with period $p_{i}$ in $\bigcup_{k=0}^{p_{i}-1} \boldsymbol{\Omega}^{j}\left(A_{i}^{k}\right)(j=0,1, \ldots, m)$.

(b) If $l$ is a divisor of $q$ then $P_{h}^{l} \cap\left(U_{i} \backslash I_{i}\right)=\varnothing$ (see Figure 2). 
Proof of Proposition 1. (a) It is easy to see (a) is implied by (A) above.

(b) Using Lemma 0 we can see that if $\left\{x_{k}\right\}_{k=0}^{l-1}$ is a periodic orbit of $h$ and $\left\{x_{k}\right\}_{k=0}^{l-1} \cap U_{i} \neq \varnothing$ then $\left\{x_{k}\right\}_{k=0}^{l-1} \subset U_{i}$. The same remains valid for $I_{i}$ because $h\left(i_{i}\right) \subset I_{i}$, thus if (b) is false then $\left\{x_{k}\right\}_{k=0}^{l-1} \subset U_{i} \backslash I_{i}$. However, since $l$ is odd $x_{0}$ and $x_{l}$ are in different components of $U_{i} \backslash I_{i}$ and thus $x_{0} \neq x_{l}$.

We now proceed to our Step 2.

\section{STEP 2}

Suppose that $\psi^{2} \in B(g, \delta)$. Let $\eta>0$ be so small that $\varphi^{2} \in B(g, \delta)$ holds for every $\varphi \in B(\psi, \eta)$. In [3, Theorem 1] it is proved that: there exists a residual subset $A$ of $C$ such that for every $\varphi \in A$ and $u, v \in \mathbb{N}$, every neighbourhood of any periodic point of $\varphi$ with period $v$ contains periodic points of $\varphi$ with period $u v$.

Choose $\varphi \in B(\psi, \eta) \cap A$ and put $h=\varphi^{2}$. Let $1 \leq i \leq n+1$ and $0 \leq j \leq m$ arbitrary. From Proposition 1 we know that $\bigcup_{k=0}^{p_{i}-1} \Omega^{j}\left(A_{i}^{k}\right)$ contains a periodic orbit $\left\{x_{k}\right\}_{k=0}^{p_{i}-1}$ of $h$ with period $p_{i}$. Since $h=\varphi^{2}$ one can easily see that either there is a periodic orbit $\left\{a_{k}\right\}_{k=0}^{2 p_{i}-1}$ of $\varphi$ with period $2 p_{i}$ such that $a_{2 k}=x_{k}$ $\left(k=0,1, \ldots, p_{i}-1\right)$ or $\left\{x_{k}\right\}_{k=0}^{p_{i}-1}$ is a periodic orbit of $\varphi$ itself. In the latter case, using the previous statement with $v=p_{i}$ and $u=2$, we get that there is a periodic orbit $\left\{a_{k}\right\}_{k=0}^{2 p_{i}-1}$ of $\varphi$ such that $\left\{a_{k}\right\}_{k=0}^{2 p_{i}-1} \subset \bigcup_{k=0}^{p_{i}-1} \Omega^{j}\left(A_{i}^{k}\right)$. Hence we have shown that

Proposition 2. For every $1 \leq i \leq n+1$ and $0 \leq j \leq m$ there exists a periodic orbit $\left\{a_{k}\right\}_{k=0}^{2 p_{i}-1}$ of $\varphi$ so that

$$
\left\{a_{2 k}\right\}_{k=0}^{p_{i}-1} \subset \bigcup_{k=0}^{p_{i}-1} \Omega^{j}\left(A_{i}^{k}\right) .
$$

Since $\left\{a_{2 k-1}\right\}_{k=0}^{p_{i}}$ is a periodic orbit of $\varphi$ with period $2 p_{i},\left\{a_{2 k-1}\right\}_{k=0}^{p_{i}}$ is a periodic orbit of $h$ with period $p_{i}$. Thus Lemma 0 implies that

$$
\left\{a_{2 k-1}\right\}_{k=0}^{p_{i}} \subset \bigcup_{z=1}^{m} W_{z} \cup \bigcup_{l=1}^{n} V_{l} \cup \bigcup_{k=1}^{n+1} U_{k} .
$$

We will show this is impossible via the four lemmas.

We use the notation of Proposition 2; clearly we may assume that $a_{2 k} \in \Omega^{j}\left(A_{i}^{k}\right)$ $\left(0 \leq k \leq p_{i}-1\right)$.

Lemma 1. $\left\{a_{2 k-1}\right\}_{k=1}^{p_{i}}$ is not contained in $U_{i}$.

Proof of Lemma 1. We actually prove more; namely,

(i) $\left\{a_{2 k-1}\right\}_{k=1}^{p_{i}}$ is not contained in $\bigcup_{k=0}^{p_{i}-1} \Omega^{j}\left(A_{i}^{k}\right)$,

(ii) $\left\{a_{2 k-1}\right\}_{k=1}^{p_{i}}$ is not contained in $\Omega^{j}\left(I_{i}\right)$ and then

(iii) $\left\{a_{2 k-1}\right\}_{k=1}^{p_{i}}$ is not contained in $U_{i}$. 
Assume first that

$$
\left\{a_{2 k-1}\right\}_{k=1}^{p_{i}} \subset \bigcup_{k=0}^{p_{i}-1} \Omega^{j}\left(A_{i}^{k}\right) .
$$

Since $\varphi\left(a_{2 p_{i}-1}\right)=a_{0}$ and $a_{0} \in \Omega^{j}\left(A_{0}^{i}\right), a_{2 p_{i}-2} \in \Omega^{j}\left(A_{p_{i}-1}^{i}\right)$ (see Figure 1) we have $\left[a_{0}, a_{2 p_{i}-2}\right] \subset \varphi\left(\left\langle a_{2 p_{i}-1}, a_{2 p_{i}-3}\right\rangle\right)$ thus $\varphi\left(\left\langle a_{2 p_{i}-1}, a_{2 p_{i}-3}\right\rangle\right)$ contains $\left\{a_{2 k-1}\right\}_{k=1}^{p_{i}}$ except for (at most) two elements. Thus $\varphi^{2}\left(\left\langle a_{2 p_{i}-1}, a_{2 p_{i}-3}\right\rangle\right) \supset$ $\left\{a_{2}, a_{4}, \ldots, a_{2 p_{i}-4}\right\}$ and this implies that

(a) $a_{2 p_{i}-1} \approx a_{2 p_{i}-2}$ or

(b) $a_{2 p_{i}-3} \approx a_{2 p_{i}-2}$ (see Figure 1),

where $x \approx y$ iff there are indices $i, j, k$ such that $x, y \in \Omega^{j}\left(A_{k}^{i}\right)$. If (a) then $a_{3} \in\left(a_{0}, a_{2 p_{i}-1}\right) \subset \varphi\left(\left\langle a_{2 p_{i}-1}, a_{2 p_{i}-2}\right\rangle\right)$ thus $a_{2} \in \varphi^{2}\left(\left\langle a_{2 p_{i}-1}, a_{2 p_{i}-3}\right\rangle\right)$ which is impossible. If (b) then $a_{1} \in\left\langle a_{2 p_{i}-2}, a_{2 p_{i}-1}\right\rangle \subset \varphi\left(\left\langle a_{2 p_{i}-3}, a_{2 p_{i}-2}\right\rangle\right)$ thus $a_{2} \in$ $\varphi^{2}\left(\left\langle a_{2 p_{i}-3}, a_{2 p_{i}-2}\right\rangle\right)$ which is impossible.

Next assume that $\left\{a_{2 k-1}\right\}_{k=1}^{p_{i}} \subset \Omega^{j}\left(I_{i}\right)$. Then from (1) we get $\left\{a_{2 k-1}\right\}_{k=1}^{p_{i}} \subset$ $I_{i}^{j}=\Omega^{j}\left(I_{i}\right) \backslash \bigcup_{k=0}^{p_{i}-1} \Omega^{j}\left(A_{i}^{k}\right)$ (see Figure 1). But $a_{2 p_{i}-1} \in I_{i}^{j} \subset\left[a_{0}, a_{2 p_{i}-2}\right] \subset$ $\varphi\left(\left\langle a_{2 p_{i}-1}, a_{2 p_{i}-3}\right\rangle\right)$ and thus $a_{4} \in \varphi^{2}\left(\left\langle a_{2 p_{i}-1}, a_{2 p_{i}-2}\right\rangle\right) \subset h\left(I_{i}^{j}\right)$ contradicting $a_{0} \approx \Omega^{j}\left(d_{0}\right)$.

Finally suppose $\left\{a_{2 k-1}\right\}_{k=1}^{p_{i}} \subset U_{i} \backslash \Omega^{j}\left(I_{i}\right)$. Since $p_{i}$ is a divisor of $q$ it follows from Proposition 1 that it is enough to show that $\left\{a_{2 k-1}\right\}_{k=1}^{p_{i}}$ is not contained in $I_{i}$. Suppose that

$$
\left\{a_{2 k-1}\right\}_{k=1}^{p_{i}} \subset I_{i} \text {. }
$$

Then there exists $0 \leq 1 \leq j$ such that $\left\{a_{2 k-1}\right\}_{k=1}^{p_{i}} \subset \Omega^{l}\left(I_{i}\right) \backslash \Omega^{j}\left(I_{i}\right)$, this follows from (ii). Since $\left\{a_{2 k-1}\right\}_{k=1}^{p_{i}}$ is a periodic orbit of $h$ with period $p_{i}$, there are odd numbers $1 \leq m_{1}, m_{2} \leq 2 p_{i}-1$ such that $h\left(a_{m_{1}}\right)>a_{m_{1}}$ and $h\left(a_{m_{2}}\right)<a_{m_{2}}$. From $(C)$ it follows that

$$
a_{2 p_{i}-4} \in\left[a_{m_{1}}, a_{m_{2}}\right] \subset \varphi\left(\left\langle a_{0}, a_{2 p_{i}-2}\right\rangle\right) .
$$

Therefore $a_{2 p_{i}-5} \in \varphi^{2}\left(\left\langle a_{0}, a_{2 p_{i}-2}\right\rangle\right) \subseteq h\left(\Omega^{j}\left(I_{i}\right) \subset \Omega^{j}\left(I_{i}\right)\right)$, which is a contradiction. This completes the proof of Lemma 1.

Lemma 2. If $j_{1} \neq j, 0 \leq j_{1} \leq m$ is fixed, and if $\left\{y_{k}\right\}_{k=0}^{p_{i}-1} \subset \bigcup_{k=0}^{p_{i}-1} \Omega^{j_{1}}\left(A_{i}^{k}\right)$ is a periodic orbit of $h$ and $\left\{b_{l}\right\}_{l=0}^{2 p_{i}-1}$ is a periodic orbit of $\varphi$ such that $y_{k}=b_{2 k} \in$ $\Omega^{j_{1}}\left(A_{i}^{k}\right) \quad\left(k=0,1, \ldots, p_{i}-1\right)$, then there do not exist indices $0 \leq j_{2}, j_{3} \leq$ $2 p_{i}-1,1 \leq j_{4} \leq m$ such that $a_{j_{2}}, b_{j_{3}} \in W_{j_{4}}$.

Proof of Lemma 2. Assume that there exists a periodic orbit $\left\{b_{l}\right\}_{l=1}^{2 p_{i}-1}$ of $\varphi$ such that $b_{2 k} \in \Omega^{j_{1}}\left(A_{i}^{k}\right) \quad\left(k=0,1, \ldots, p_{i}-1\right)$ and $a_{j_{2}}, b_{j_{3}} \in W_{j_{4}}$. We can assume $j<j_{1}$. Then it follows from Lemma 0 that there are indices $j_{5}, j_{6}$ such that $0 \leq j_{5} \leq 2 p_{i}-1,1 \leq j_{6} \leq m$ and such that $a_{1}, b_{j_{5}} \in W_{j_{6}}$. 
Thus, since $\varphi\left(a_{1}\right)=a_{2}$ and $\varphi\left(b_{j_{5}}\right) \in \Omega^{j_{1}}\left(I_{1}\right)$, the point $a_{4}$ belongs to $\varphi\left(\left\langle a_{1}, b_{j_{5}}\right\rangle\right)$ and this implies $a_{5}$ is an element of $\varphi^{2}\left(\left\langle a_{1}, b_{j_{5}}\right\rangle\right) \subset h\left(W_{j_{6}}\right)$. But $a_{1} \in W_{j_{6}}$ which implies that $a_{3} \in h\left(W_{j_{6}}\right)$ and $a_{5} \in h^{2}\left(W_{j_{6}}\right)$. Thus $h^{2}\left(W_{j_{6}}\right) \cap h\left(W_{j_{6}}\right) \neq \varnothing$ and this contradicts Lemma 0.

It should be noted that Lemma 2 implies that there exists $0 \leq 1 \leq m$ so that for every periodic orbit $\left\{b_{k}\right\}_{k=0}^{2 p_{i}-1}$ of $\varphi$ if $b_{2 k} \in \Omega^{l}\left(A_{k}^{i}\right) \quad\left(k=0,1, \ldots, p_{i}-1\right)$, then $\left\{b_{2 k-1}\right\}_{k=0}^{2 p_{i}-1} \cap \bigcup_{z=0}^{m} W_{z}=\varnothing$.

Lemma 3. $\left\{a_{2 k-1}\right\}_{k=1}^{p_{i}-1}$ is not contained in $U_{i_{1}}$ where $1 \leq i_{1} \leq n+1$ and $i_{1} \neq i$. Proof of Lemma 3. Assume that $\left\{a_{2 k-1}\right\}_{k=1}^{p_{i}} \subset U_{i_{1}}$. First we suppose that $\left\{a_{2 k-1}\right\}_{k=1}^{p_{i}}$ is not contained in $\Omega^{m+1}\left(I_{i_{1}}\right)$. Then there exists $0 \leq w \leq m$ such that

$$
\left\{a_{2 k-1}\right\}_{k=1}^{p_{i}} \subset \Omega^{w}\left(I_{i_{1}}\right) \backslash \Omega^{w+1}\left(I_{i_{1}}\right) .
$$

Assume that $\left\{b_{l}\right\}_{l=0}^{2 p_{i}-1}$ is a periodic orbit of $\varphi$ such that $b_{2 l} \in \Omega^{w}\left(A_{l}^{i_{1}}\right)$ for every $0 \leq 1 \leq p_{i_{1}}-1$. Then

$$
b_{2 p_{i_{1}}-4} \in \varphi\left(\left\langle a_{0}, a_{2 p_{i}-2}\right\rangle\right)
$$

(this follows from property (C) of $\left.\Phi_{(I)}^{p}\right)$. Thus

$$
b_{2 p_{i_{1}}-3} \in \varphi^{2}\left(\left\langle a_{0}, a_{2 p_{i}-2}\right\rangle\right) \subset \Omega^{j}\left(I_{i}\right)
$$

and hence that

$$
\left\{b_{2 l-1}\right\}_{l=1}^{p_{i}-1} \subset \Omega^{j}\left(I_{i}\right) \backslash \bigcup_{k=0}^{p_{i}-1} \Omega^{j}\left(A_{k}^{i}\right)=I_{i}^{j}
$$

because $p_{i_{1}} \neq p_{i}$ and $p_{i}, p_{i_{1}}$ are prime numbers. Further

$$
a_{2 p_{i}-1} \in\left\langle b_{0}, b_{2 p_{i_{1}}-2}\right\rangle \subset \varphi\left(\left\langle b_{2 p_{i_{1}}-1}, b_{2 p_{i_{1}}-3}\right\rangle\right)
$$

and thus $a_{0} \in \varphi^{2}\left(\left\langle b_{2 p_{i_{1}}-1}, b_{2 p_{i_{1}}-3}\right\rangle\right)$. This is a contradiction since $a_{0} \notin \varphi^{2}\left(I_{i}^{j}\right)$ (see Figure 1).

Next suppose that $\left\{a_{2 k-1}\right\}_{k=1}^{p_{i}} \subset \Omega^{m+1}\left(I_{i_{1}}\right)$ (see Figure 3 ). Then for every $0 \leq z \leq m$ we choose a periodic orbit of $\varphi,\left\{b_{l}^{z}\right\}_{l=0}^{2 p_{i_{1}}-1}$, so that

$$
b_{2 l}^{z} \in \Omega^{z}\left(A_{l}^{i_{1}}\right) \quad\left(l=0,1, \ldots, p_{i_{1}}-1\right) .
$$

It follows from Lemma 2 that there exists $0 \leq z_{0} \leq m$ such that $\left\{b_{2 l-1}^{z_{0}}\right\}_{l=1}^{p_{i}}$ does not intersect any $W_{l}$. Since $\left\{b_{2 l-1}^{z_{0}}\right\}_{l=1}^{p_{i}}$ is a periodic orbit of $h$, it intersects some $U_{k}$ or $V_{k}$. Since $h\left(\Omega^{j+1}\left(I_{i}\right)\right)$ does not contain $a_{2 l},\left(l=0, \ldots, p_{i}-\right.$ 1), we get from Lemma 0 and the assumption $\left\{a_{2 k-1}\right\}_{k=1}^{p_{i}} \subset \Omega^{m+1}\left(I_{i_{1}}\right)$, that $\left\{b_{2 l-1}^{z_{0}}\right\}_{l=1}^{p_{i}} \subset I_{i} \backslash \Omega^{j+1}\left(I_{i}\right)$. But since $j \leq m$ an analogous case with changing 


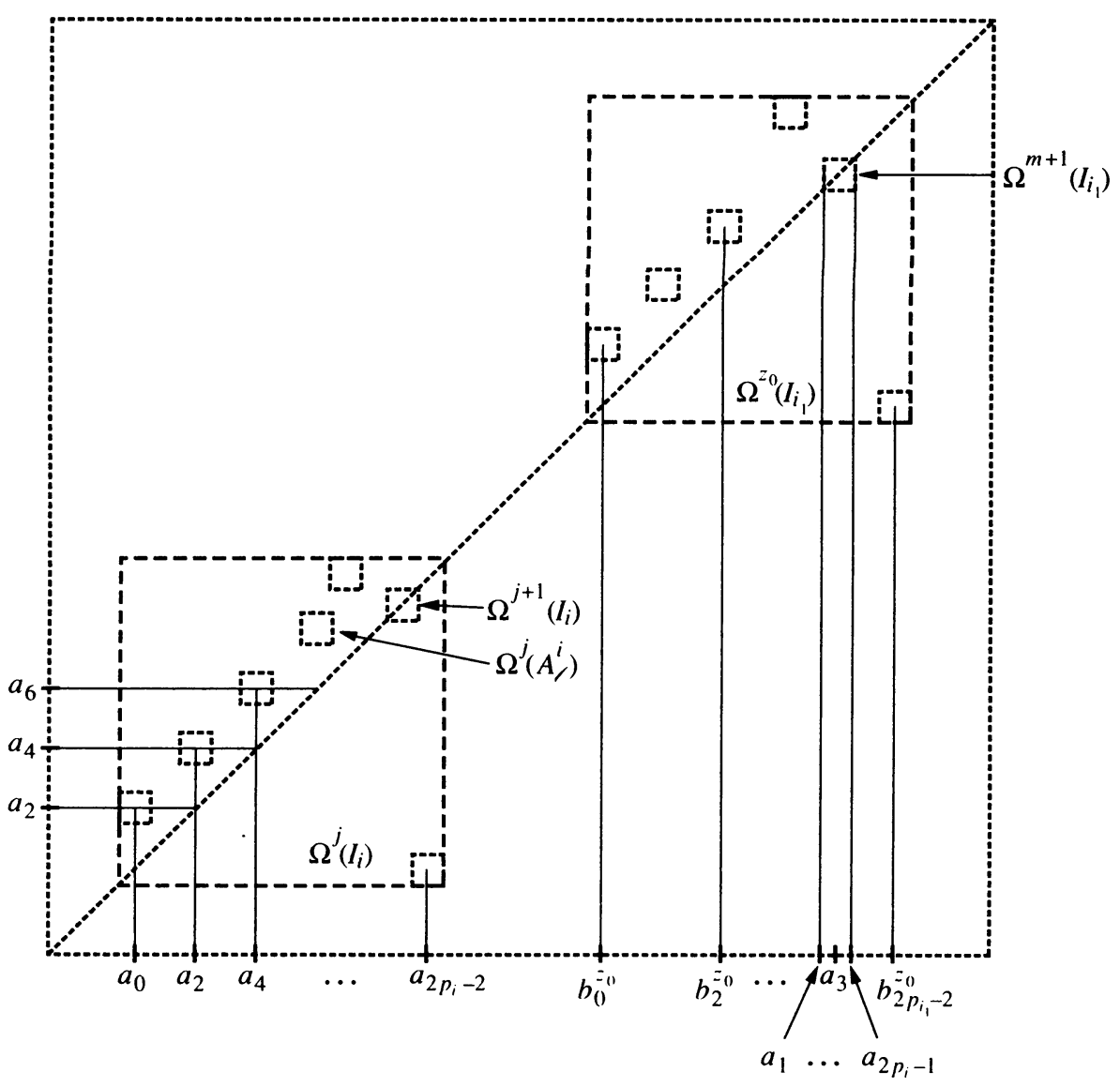

FIGURE 3

rules between $\left\{a_{k}\right\}_{k=0}^{2 p_{i}-1}$ and $\left\{b_{l}^{z_{0}}\right\}_{l=0}^{2 p_{i_{1}}-1}$ was investigated in the first part of this proof showing that this is impossible.

We shall write $U_{i} \sim V_{j}$ if there is a periodic orbit $\left\{a_{k}\right\}_{k=0}^{2 p_{i}-1}$ of $\varphi$ such that $\left\{a_{2 l}\right\}_{l=0}^{p_{i}-1} \subset U_{i}$ and $\left\{a_{2 l-1}\right\}_{l=1}^{p_{i}} \subset V_{j}$. Lemmas 1,2 , and 3 imply that for every $1 \leq i \leq n+1$ there exists $1 \leq j \leq n$ such that $U_{i} \sim V_{j}$.

Lemma 4. There exist indices $i, j, k$ such that $1 \leq i \leq j<k \leq n+1$, with $U_{i} \sim V_{j}$ and $U_{k} \sim V_{j}$.

Proof of Lemma 4. Since the number of the $U_{j}$ 's is greater than the number of the $V_{j}$ 's, there exist $i, j$ and $k$ such that

$$
U_{i} \sim V_{j}, \quad U_{k} \sim V_{j} .
$$

If the desired condition: $(i \leq j<k)$, does not hold for these $i, j$ and $k$, we can assume that $i<k \leq j$. (The situation when $j<i, k$ is similar). Then we claim there is no $1 \leq l \leq n+1$ such that $V_{i} \backsim U_{l}$. For if $V_{i} \backsim U_{l}$ then, 
since $\varphi\left(V_{j}\right) \cap U_{i} \neq \varnothing$ and $\varphi\left(V_{j}\right) \cap U_{k} \neq \varnothing$, we would get $\varphi\left(V_{j}\right) \supset V_{i}$. Thus $\varphi^{2}\left(V_{j}\right) \cap U_{l} \neq \varnothing$ which contradicts Lemma 0 . Therefore deleting both $U_{i}$ and $V_{i}$ the remaining $U_{j}$ 's and $V_{j}$ 's still have the property that for each $j$ there is a $k$ with $U_{j} \backsim U_{k}$.

We continue with this process of checking the condition and deleting until we have either the condition fulfilled or have deleted down to two $U$ 's and only one $V$. Then the desired condition will hold.

Let $i, j, k$ be as in Lemma 4. Then, since $i \leq j<k$, we have both $\varphi\left(V_{j}\right) \cap$ $U_{i} \neq \varnothing$ and $\varphi\left(V_{j}\right) \cap U_{k} \neq \varnothing$ and $\varphi\left(V_{j}\right) \supset V_{j}$. This implies $\varphi^{2}\left(V_{j}\right) \cap U_{i} \neq \varnothing$ and $\varphi$ in turn contradicts Lemma 0 . This completes the proof of the theorem.

\section{ACKNOWLEDGMENT}

The author would like to thank professor Miklós Laczkovich for his useful advice.

\section{REFERENCES}

1. P. D. Humke and M. Laczkovich, On the squares of continuous functions, Ergodic Theory Dynamical Systems (to appear).

2. 32 (1989), 483-494.

3. K. Simon, On the periodic points of a typical continuous function, Proc. Amer. Math. Soc. 105 (1989), 244-249.

4. $\longrightarrow$ Some dual statements concerning Wiener measure and Baire category, Proc. Amer. Math. Soc. 106 (1989), 455-463.

5. __ Typical continuous functions are not iterates, Acta Math. Hungar (to appear).

Department of Mathematics, Technical University, Miskolc, Hungary 